\title{
Micro Sensors: Linking Real-Time Oscillatory Shear Stress with Vascular Inflammatory Responses
}

\author{
Tzung K. Hsiai, ${ }^{1}$ Sung K. Cho, ${ }^{4}$ PaK K. Wong, ${ }^{2}$ Michael H. Ing, ${ }^{1}$ Adler Salazar, ${ }^{1}$ Susan Hama, ${ }^{3}$ \\ MOHAMAd NAVAB, ${ }^{3}$ Linda L. DEMER, ${ }^{3}$ and CHIH-Ming HO ${ }^{2}$

\begin{abstract}
${ }^{1}$ Department of Biomedical Engineering and Division of Cardiovascular Medicine, USC School of Engineering and School of Medicine, Los Angeles, CA; ${ }^{2}$ Department of Mechanical and Aerospace Engineering, UCLA School of Engineering and Applied Sciences, Los Angeles, CA; ${ }^{3}$ Division of Cardiology, Department of Medicine, UCLA School of Medicine, Los Angeles, CA; and ${ }^{4}$ Department of Mechanical Engineering, University of Pittsburgh, Pittsburgh, PA 15261
\end{abstract}

(Received 6 December 2002; accepted 4 October 2003)

\begin{abstract}
The important interplay between blood circulation and vascular cell behavior warrants the development of highly sensitive but small sensing systems. The emerging micro electro mechanical systems (MEMS) technology, thus, provides the high spatiotemporal resolution to link biomechanical forces on the microscale with large-scale physiology. We fabricated MEMS sensors, comparable to the endothelial cells (ECs) in size, to link real-time shear stress with monocyte/EC interactions in an oscillatory flow environment, simulating the moving and unsteady separation point at arterial bifurcations. In response to oscillatory shear stress $(\tau)$ at $\pm 2.6 \mathrm{dyn} / \mathrm{cm}^{2}$, time-averaged shear stress $\left(\tau_{\text {ave }}\right)=0$ at $0.5 \mathrm{~Hz}$, individual monocytes displayed unique to-and-fro trajectories, undergoing rolling, binding, and dissociation with other monocyte, followed by solid adhesion on EC. Incorporating with cell-tracking velocimetry, we visualized that these real-time events occurred over a dynamic range of oscillating shear stress between \pm 2.6 $\mathrm{dyn} / \mathrm{cm}^{2}$ and Reynolds number between 0 and 22.2 in the presence of activated adhesion molecule and chemokine mRNA expression.
\end{abstract}

Keywords-Micro electro mechanical systems (MEMS), Celltracking velocimetry, Shear stress, Endothelial cells, Monocytes.

\section{INTRODUCTION}

The endothelium, which lines the inner lumen of blood vessel walls, is intimately involved in the recruitment of leukocytes fundamental to the initiation of immune responses. ${ }^{24,36}$ The process of leukocyte adhesion to endothelial cells (ECs) involves a complex balance of forces arising from hydrodynamic shear effects and the dynamics of leukocyte/EC binding. Shear stress, the tangential drag force of blood passing along the surface of the endothelium, ${ }^{7}$ imparts profound effects on EC function. ${ }^{4,6,10}$ Around arterial bends and branches in which the inflammatory responses prevail, the fluid mechanical

Address correspondence to T. K. Hsiai, MD, PhD, Department of Biomedical Engineering and Division of Cardiovascular Medicine, University of Southern California, Olin Hall of Engineering, 530A, Los Angeles, CA 90089-1451. Electronic mail: thsiai@usc.edu environment is distinct from the laminar pulsatile environment present in the long, straight sections of the vessel wall. Oscillatory flow with a time-averaged shear stress of 0 , characteristic of reattachment points in the arterial branches, modulates the biological activities of EC. ${ }^{20,29,39}$

Leukocyte rolling, adhesion, and transmigration have been observed in response to inflammatory stimuli at the microvascular levels. ${ }^{30}$ King and Hammer have numerically examined leukocyte-leukocyte interactions during rolling. ${ }^{17}$ What is missing is a dynamic, high spatial, and temporal resolution view of these events in a more complex physiologic flow environment simulating reattachment points at arterial bifurcations. ${ }^{8}$ Therefore, we have developed specialized micro electro mechanical systems (MEMS) sensors and cell-tracking velocimetry to visualize monocyte/EC binding kinetics in the presence of oscillatory shear stress with high spatial and temporal resolution.

Despite evidence for direct correlations of shear stress with distribution of focal atherosclerotic lesions and the computational fluid dynamic (CFD) determinations of shear stress in the arterial circulation, ${ }^{19,27,34}$ no direct measurement has been achieved to resolve the spatial and temporal variations. The challenge is to acquire an accurate near-wall shear stress. When a hot wire is placed very close to the wall, the surface affects the heat transfer; thus, decreasing the sensitivity. The noise level of optical velocimetry, such as obtained from a laser Doppler velocimeter or a particle image velocimeter, increases mostly because of the reflection from the wall. MEMS, which are derived from semiconductor technology, enable the realization of highly miniaturized shear stress sensors, nearly the size of an elongated EC. The heat transfer from a resistively heated element to the flowing fluid can be measured as changes in voltage $(V)$, allowing for the determination of shear stress levels from a linear relation between $V^{2}$ and $\tau^{1 / 3}$. ${ }^{22}$ The sensor's small dimension offers the possibility to measure shear stress in various arterial geometries under time-varying nature of pulsatile flow. 
By simulating oscillatory flow at the unsteady and moving separation point of arterial bifurcations, ${ }^{26}$ we were able to demonstrate the unique trajectory patterns of monocyte/EC binding kinetics in response to real-time shear stress at $\pm 2.6 \mathrm{dyn} / \mathrm{cm}^{2}, 0.5 \mathrm{~Hz}$, and time-averaged shear stress $\left(\tau_{\text {ave }}\right)=0$. Individual cell-cell interaction events were visualized in the presence of mRNA expression of adhesion molecules and chemokines.

\section{APPARATUS AND METHODS}

\section{MEMS Shear Stress Sensors Operating Principles}

The operation of the shear stress sensor is based on the fully developed flow condition in which the rate of heat loss from a heated resistive element to the fluid-flow is dependent on the boundary-layer velocity profile. ${ }^{9}$ The change in temperature of the local flow milieu leads to the change in resistivity of the sensors.

The dynamic performance of the sensors is characterized by a three-layer structure [Fig. 1(a)]: (1) the sensing element or the film layer is the top layer; (2) the silicon-nitride diaphragm in the middle serves as an insulation layer; and (3) the silicon substrate at the bottom provides heat sink. From Fig. 1(a), the energy balance equation is expressed as ${ }^{15}$

$$
P=I^{2} R=c_{\mathrm{f}} m_{\mathrm{f}} \frac{d T_{\mathrm{f}}}{d t}+c_{\mathrm{i}} m_{\mathrm{i}} \frac{d T_{\mathrm{i}}}{d t}+h\left(u_{\tau}\right) \mathbf{A}\left(T-T_{0}\right),
$$

where $h$ denotes the heat-transfer coefficient. The subscripts $\mathrm{f}, \mathrm{i}$, and $\mathrm{c}$ represent the convective heat transfer from the film and insulation layer to the fluid, respectively. The term " $c$ " is defined as specific heat and " $m$ " as mass. $T$ is the temperature of the sensor element and $T_{0}$ the heat sink. "A" denotes the cross-sectional area of the sensor as $\mathbf{A}=W L$, where $W$ and $L$ represent the width and length. The convective heat-transfer coefficient is represented by $h\left(u_{\tau}\right)$, which is a function of shear velocity, denoted as $u_{\tau}$. The relation between the shear velocity and the wall shear stress is $\tau_{w}=u_{\tau}^{2} \rho$, where $\rho$ is the measured fluid density. The product of the square of current, $I^{2}$, and the resistor sensor, $R$, gives rise to the heating power, $P$, as $I^{2} R$. At a low frequency of cardiac cycles, the two unsteady terms in Eq. (1) are negligible. Thus, Eq. (1) can be simplified to set power dissipation equal to the convective heat-transfer term (from the heated sensor to the ambient fluid). The heating power for the sensor is related to the wall shear stress as ${ }^{9}$

$$
P=h\left(u_{\tau}\right) \mathbf{A}\left(T-T_{0}\right)=\left(A+B \tau^{\frac{1}{3}}\right)\left(T-T_{0}\right),
$$

where $A$ and $B$ are calibration constants. The resistance, $R$, of the semiconductor-sensing element is a function of change in temperature:

$$
R=R_{0}\left[1+\alpha\left(T-T_{0}\right)\right]
$$

where $R_{0}$ is the resistance at room temperature $T_{0}$ and $\alpha$ is the temperature coefficient of resistance (TCR). An important feature governing the thermal shear stress sensor (a)

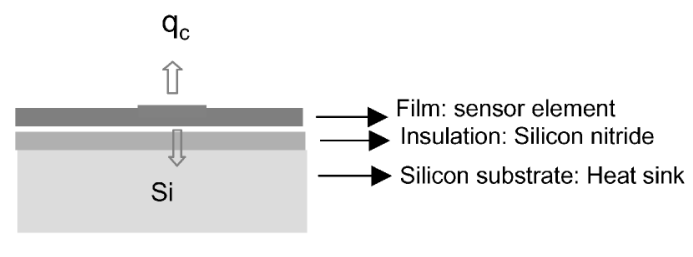

(b)

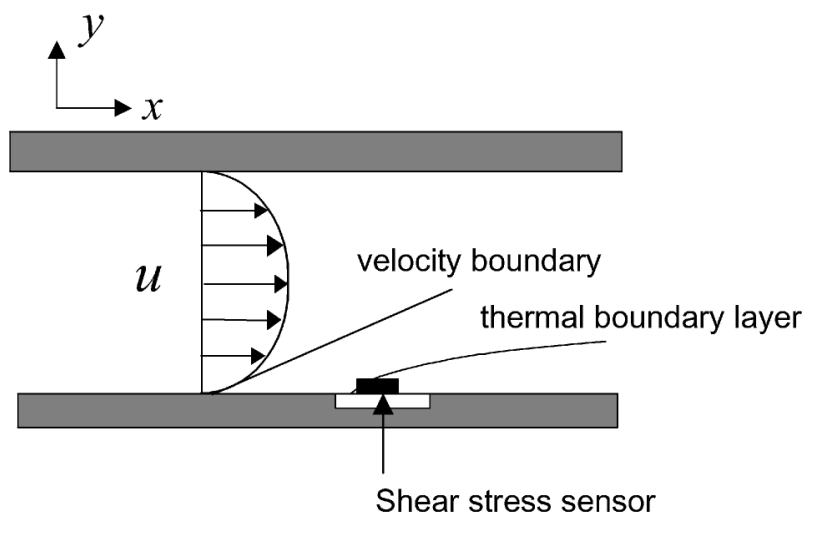

$$
\begin{gathered}
\tau_{w}=\left.\mu \frac{\partial u}{\partial y}\right|_{\text {wall }} \\
V_{o}^{2} / R_{\text {sensor }} \cong Q_{\text {convection }} \propto \tau_{w}^{1 / 3}
\end{gathered}
$$

FIGURE 1. Principle of thermal shear stress sensors. (a) Threelayer model for heat transfer with " $q_{c}$ " referring to convective heat transfer. (b) The thermal element resides within a velocity boundary layer. Thus, the rate of heat loss from a heated resistive element to the fluid flow is dependent on the velocity profile in the boundary layer. A linear relation is obtained as $V^{2} R \propto \tau^{1 / 3}$.

operation is the temperature overheat ratio, $\alpha_{T}$, defined as the relative change of sensor temperature compared with the ambient temperature:

$$
\alpha_{T}=\frac{T-T_{0}}{T_{0}}
$$

It is also commonly defined as resistance overheat ratio as, $\alpha_{R}$, which reflects the relative change of sensor resistance compared with the resistance at the ambient temperature,

$$
\alpha_{R}=\frac{R-R_{0}}{R_{0}}
$$

The resistance over heat ratio was set at 0.12 in order to minimize natural convection by the heating of the resistive element. It has been demonstrated by Huang et al. that the higher the over heat ratio, the higher the sensitivity. ${ }^{14,15}$ 
The MEMS sensors are operated by either constant temperature (CT) or constant current (CC) driving circuit. For CT mode, the sensor resistance, $R$, is kept constant by a Wheatstone bridge feedback circuit. The power can be expressed as $P=V^{2} / R$, where $V$ is the voltage across the sensor and $R$, the sensor resistance, Eq. (2) becomes

$$
V^{2} / R=\left(A_{T}+B_{T} \tau^{1 / 3}\right) .
$$

The TCR (or $\alpha$ in Eq. (3)) can be adjusted by changing the doping concentration of boron into the polysilicon strip. In a fully developed channel flow, the shear stress determines the rate of heat transfer from a heated resistive element to the surrounding fluid field. The heating power is proportional to convective heat transfer. The thickness of thermal boundary layer is likely to be smaller than that of velocity boundary layer. Thus, a linear relation between $V^{2}$ and $\tau_{\mathrm{w}}^{1 / 3}$ is established [Fig. 1b].

\section{Unique Aspects of MEMS Shear Stress Sensor Fabrication}

The schematic diagram of the sensor (Fig. 2) features the polysilicon as the heating and sensing element across the center of a cavity diaphragm. The polysilicon, measuring $2-\mu \mathrm{m}$ wide, $0.5-\mu \mathrm{m}$ high, and $80-\mu \mathrm{m}$ long, was uniformly doped with boron at $10^{16} / \mathrm{cm}^{2}$ at $60 \mathrm{keV}$ of energy to give rise to a positive TCR of $0.1 \% /{ }^{\circ} \mathrm{C}$ and a typical sheet-resistance of $50 \Omega / \square,{ }^{15}$ or a resistance between 1.25 and $5 \mathrm{k} \Omega$ at room temperature. This resistance was higher than that of the traditional metal sensor $(5-50 \Omega)$. After doping, the wafer was annealed at $1000^{\circ} \mathrm{C}$ to activate the dopant and to reduce the intrinsic stress on the polysilicon.
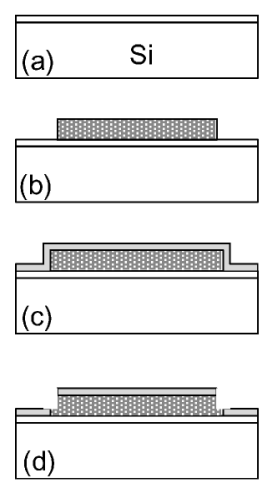

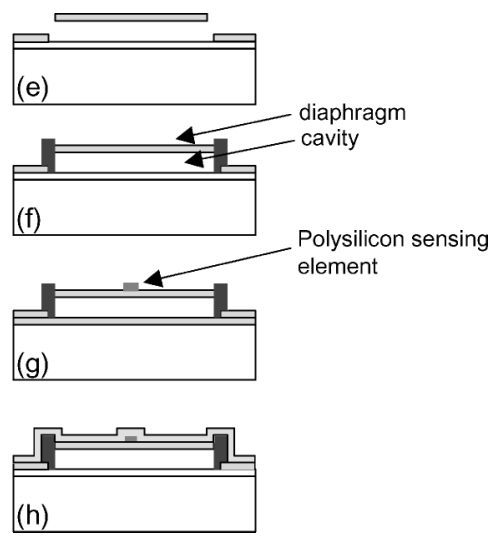

FIGURE 2. Fabrication steps of the micro thermal shear stress sensor: (a) thermal oxidation and Si3N4 deposition; (b) polysilicon deposition and patterning; (c) thermal oxidation and Si3N4 deposition; (d) opening etching holes; (e) removing the sacrificial poly-Si layer; (f) blocking the etching holes; (g) polySi deposition, ion-implantation with boron and patterning; (h) aluminum deposition and pattering for electrodes, and $\mathrm{SiO}_{2}$ deposition for water proof. A vacuum was created to optimize convective heat transfer from the heated sensor to the ambient fluid. Refer to Fig. 4(b) for the photos of sensors.
The resistance of the sensing element could be adjusted by changing the doping levels.

An array of resistors was fabricated on a single chip. The individual sensing elements were lying across the diaphragm [Fig. 3(b)]. ${ }^{12}$ The diaphragm was separated from the bottom of the cavity by an approximately $2-\mu \mathrm{m}$ gap. The pressure inside the cavity was equal to 300 mtorr $(0.04 \mathrm{~Pa})$, at which the oxide layer $(1.2 \mu \mathrm{m})$ and silicon-nitride layer $(300 \mathrm{~nm})$ were deposited in Fig. 2(f). Thermal evaporation of the aluminum metalization $(300 \mathrm{~nm}$ in height and $10 \mu \mathrm{m}$ in width) formed the leads connecting the polysilicon resistor or the sensing element to the external electronics. The vacuum cavity allowed for effective thermal isolation between the heated element and the substrate, thereby minimizing the heat conduction from the diaphragm to the substrate through the gap. ${ }^{15}$

\section{MEMS Sensor Calibration}

Calibration was performed for each individual device to establish a linear relation between $V^{2}$ and $\tau_{\mathrm{w}}^{1 / 3}$. We generated four different pulsatile flows with various mean flow rates, $Q_{n}$ [Fig. 4(a)]. ${ }^{4}$ The average shear stress values corresponding to these individual flow rates were obtained by using the equation for Newtonian fluid as

$$
\tau_{\mathrm{w}}=\left(\frac{6 \mu}{h^{2} w}\right) Q_{n}
$$

where $w$ denotes the width of channel and $\mu$ the dynamic viscosity of fluid. The voltages and the corresponding shear stresses were obtained from Eq. (6). ${ }^{22}$ A linear relation between $V^{2}$ and $\tau^{1 / 3}$ were established by plotting the averaged voltage output signals $\left(V_{n}\right)$ with the corresponding values in mean shear stress at $37 \pm 0.15^{\circ} \mathrm{C}$ [Fig. 4(b)].

\section{Interfacing MEMS Sensors with a Pulsatile Flow Channel}

A pulsatile flow system was used to deliver well-defined flow profiles simulating the flow conditions in the arterial circulation (Fig. 3). ${ }^{11}$ This unique configuration ensured velocity uniformity and absence of flow separation across the width of the channel during flow reversal. Because of the symmetry of the rectangular flow channel, we were able to flush-mount the sensor opposite to the EC monolayers [Fig. 3(a)], which were seeded on the bottom parallel plate. This approach circumvented the local flow disturbance introduced by conventional probes.

\section{Validation of Real-Time Shear Stress}

Shear stress was also obtained on the basis of the Bernoulli's theorem. Using an orifice located upstream from the flow channel, we recorded the pressure signals with a piezoelectric sensor at $10 \mathrm{~Hz}$ (SenSym Model 143SC03D). The signals were phase-averaged and then, converted to the 


\section{Calibration at Q1, Q2, Q3, and Q4}

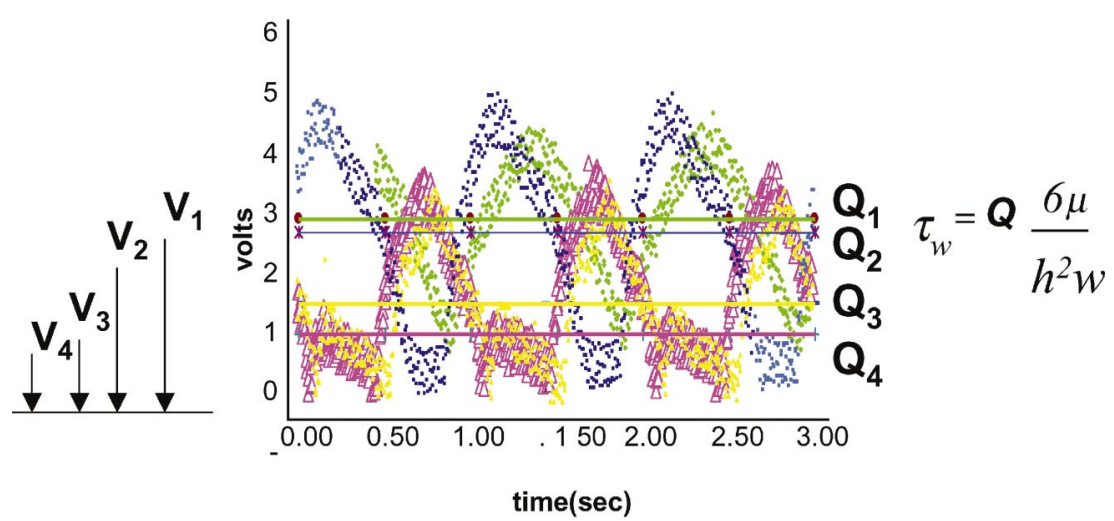

(a)

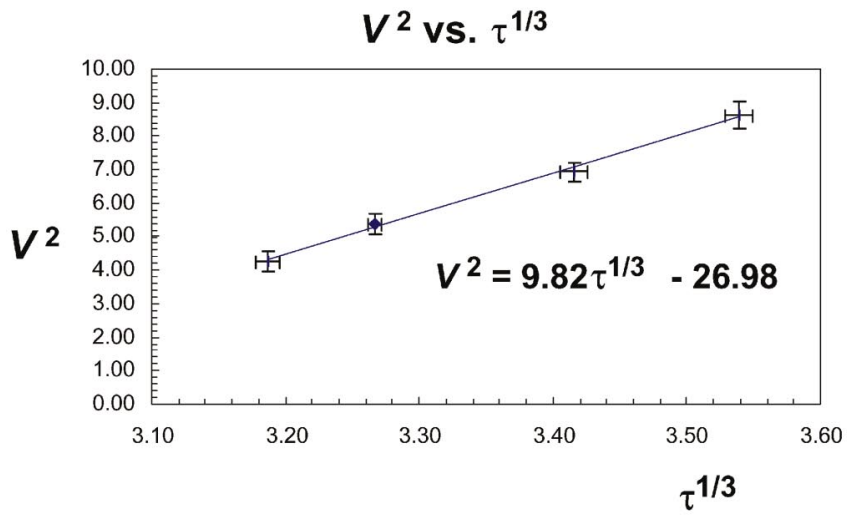

(b)

FIGURE 3. (a) The MEMS sensor array was flush-mounted on the upper wall of the pulsatile flow channel. Confluent BAEC monolayers were seeded on the bottom. (b) A photograph of individual shear stress sensors illustrates the polysilicon as a sensing element. The diaphragm [refer to Fig. 2(f)] was bent down by the external atmospheric pressure giving rise to an optical interference patterns noted as Newton rings.

pulsatile flows (Fig. 5). The shear stress was expressed as ${ }^{13}$

$$
\tau_{\mathrm{w}}(t)=\left.\mu \frac{\partial u}{\partial y}\right|_{y=h / 2}=-u_{n} \frac{\lambda_{n} \mu}{\alpha_{n} h \sqrt{i}} \tanh \left(\frac{\alpha_{n} \sqrt{i}}{2}\right) e^{i n \varpi t}
$$

where $\alpha_{n}=h \sqrt{\frac{n \varpi}{v}}$, Womersley number for a given angular frequency $(n \varpi)$, and $\lambda_{n}=\frac{P_{n} h^{2}}{\rho u_{n} v}$, a nondimensional magnitude of the imposed sinusoidal pressure gradient varying in time with an angular frequency, $n \varpi, u_{n}$, the representative axial velocity, and $P_{n}$, the representative pressure difference. " $i$ " denotes the complex number $\left(i^{2}=-1\right)$, and $v$ the kinematic viscosity. The detailed derivation can be accessed on-line at http:// ojps.aip.org/dbt/dbt.jsp?KEY=ABMECF \&Volume $=30 .{ }^{11}$

\section{Monocyte-Tracking Velocimetry}

We developed a cell-tracking velocimetry algorithm to reconstruct the trajectories of monocyte/EC interactions in response to oscillatory shear stress. ${ }^{28}$ The time-dependent positions of monocyte/monocyte and monocyte/EC binding kinetics were recorded on videotape by a CCD camera and digitalized with a video capture card (Truevision TARGA 1000). The digital images were then analyzed by the velocimetry algorithms to capture monocyte in locomotion on the EC monolayers. The algorithm initiated the identification of monocytes by dividing the fields of interest into interrogation areas that were comparable to the size of individual monocytes. The fields of interest were sampled such that $50 \%$ of the interrogation areas were overlapped to satisfy the Nyquist sampling criteria. ${ }^{32}$ The individual monocytes and their center positions were determined by the convolution of the interrogation area [Fig. 6(a)]. The displacement of the monocytes was extracted by $2 \mathrm{D}$ crosscorrelation of the image area with the corresponding area in the next digital image. The 2D cross-correlation $F$ was defined as ${ }^{32}$

$$
F\left(D_{x}, D_{y}\right)=\iint I(x, y) I\left(x+D_{x}, y+D_{y}\right) d x d y
$$




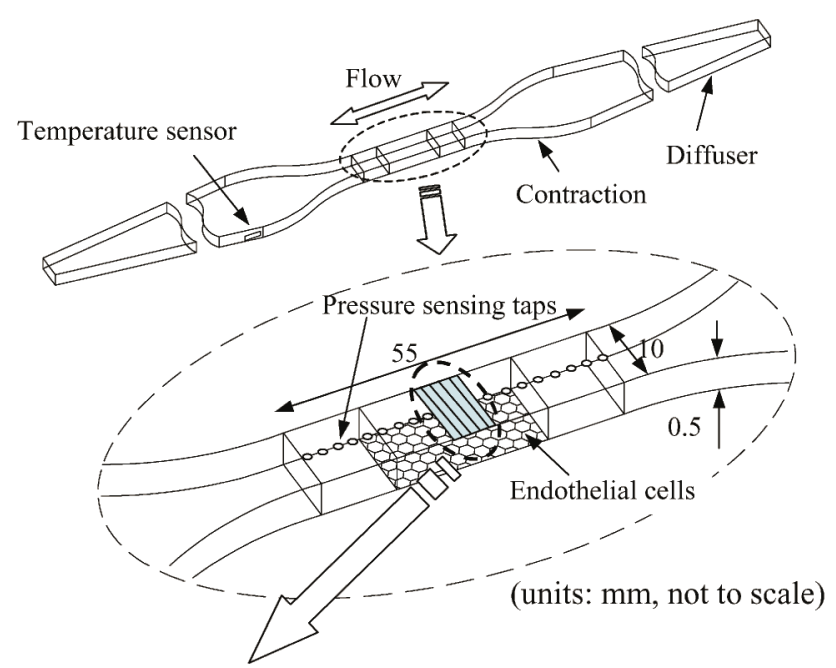

(a)
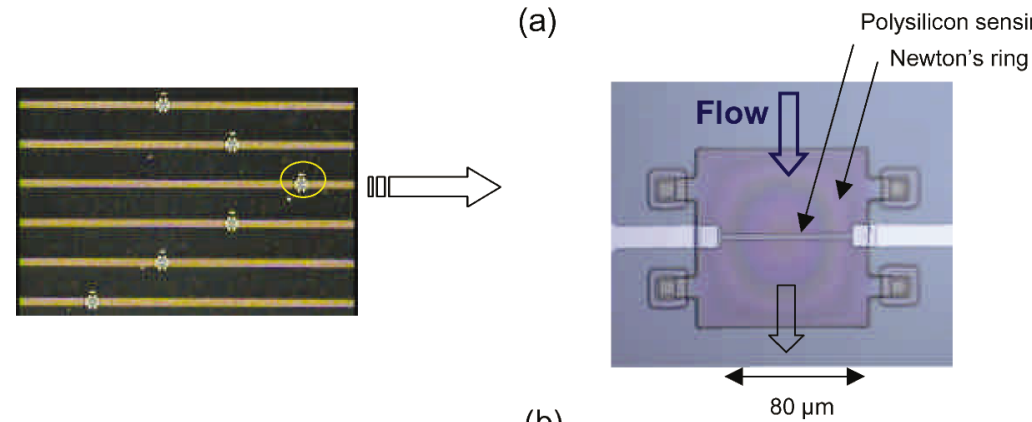

(b)

FIGURE 4. (a) Calibration was performed by obtaining the voltage signals detected by the MEMS sensors at $37 \pm 0.15^{\circ} \mathrm{C}$. Pulsatile flow profiles at four different mean flow rates $(Q)$ were recorded as voltage signals from the MEMS sensor. The mean voltage values are shown as the linear horizontal lines corresponding to the specific flow rates. (b) Linear relationship was established between the mean voltage signals and shear stress. This linear calibration curve was constructed from the four coordinates, $\left(V_{n}, \tau_{n}\right)$. This linear relationship allowed for converting voltage signal output to shear stress. The calibration constants are $A=9.82$ and $B=-26.98$

where $I$ is the intensity of the image, and $D_{x}, D_{y}$, the displacement in the $x$ and $y$ directions, respectively. The peaks of the 2D cross-correlation signal provided the displacement information of monocytes between different digital images [Fig. 6(b)].

\section{Endothelial Cell Culture}

Bovine aortic endothelial cells (BAEC) between passages 5 and 9 were seeded on Cell-Tak cell adhesive (Becton Dickson Labware, Bedford, MA) and vitrogen (Cohesion, Palo Alto, RC 0701) coated glass slides $(1 \times 5 \mathrm{~cm})$ at $3 \times 10^{6}$ cells per slide. BAEC were then grown to confluent monolayers in DMEM (Dulbecco's modified Eagle's medium) supplemented with $20 \%$ fetal bovine serum and $0.05 \%$ amphotericin $\mathrm{B}$ and $100 \mathrm{U} / \mathrm{ml}$ streptomycin for $48 \mathrm{~h}$ in $5 \% \mathrm{CO}_{2}$ at $37^{\circ} \mathrm{C}$.

\section{Experimental Protocols}

Confluent BAEC monolayers grown on the glass slide were placed in the flow channel and exposed to one of the two flow conditions at $1 \mathrm{~Hz}$ for $4 \mathrm{~h}$ : (1) pulsatile flow at a shear stress slew rate $(\partial \tau / \partial t)$ of $293 \mathrm{dyn} /\left(\mathrm{cm}^{2} \mathrm{~s}\right)$, with a time-averaged shear stress $\left(\tau_{\text {ave }}\right)$ of $50 \mathrm{dyn} / \mathrm{cm}^{2}$ [Fig. 7(a)], (2) oscillating flow $\left( \pm 2.5 \mathrm{dyn} / \mathrm{cm}^{2}\right.$ at $\left.0.5 \mathrm{~Hz}\right)$ with $\tau_{\text {ave }}=0 \mathrm{dyn} / \mathrm{cm}^{2}$ at $0.5 \mathrm{~Hz}$ [Fig. 7(b)]. For oscillating flow, minimal forward flow at a mean shear stress of $0.2 \mathrm{dyn} / \mathrm{cm}^{2}$ was provided every hour to deliver nutrients and remove waste products from the cells. The control samples were under static condition without flow exposure.

\section{Visualizing Monocyte/Endothelial Cell Interactions in Response to Oscillatory Flow}

Monocytes were isolated using a modification of the Recalde method as previously described from normal volunteers with institutional review board approval. ${ }^{5}$ Freshly isolated monocytes $\left(10^{5}\right.$ monocytes $\left./ \mathrm{cm}^{3}\right)$ were introduced into the testing channel under oscillatory flow with parameters as described in the Experimental Protocol section. The flow channel was mounted on an inverted light microscope for a real-time monitoring of cell-cell interactions. Five percent $\mathrm{CO}_{2}$ was delivered to the circulating culture medium. Monocytes and BAEC interactions in 


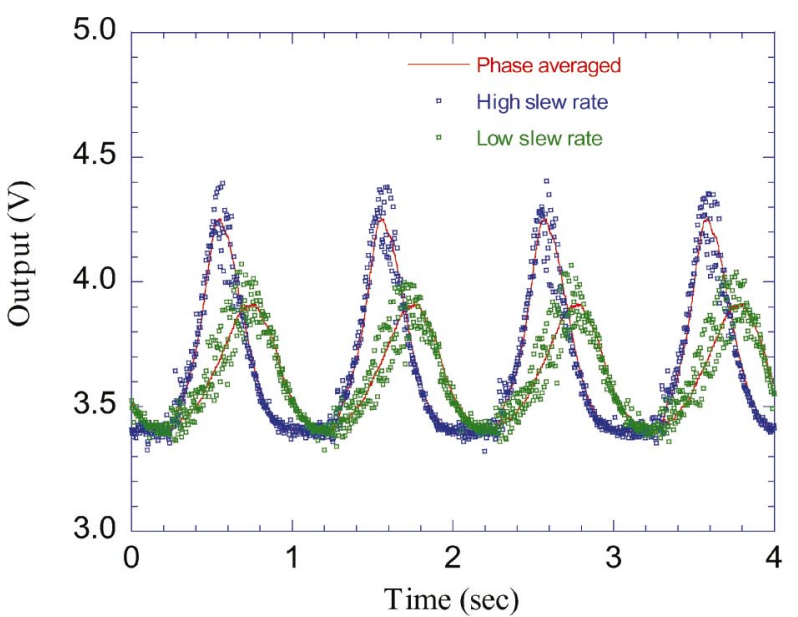

(a)

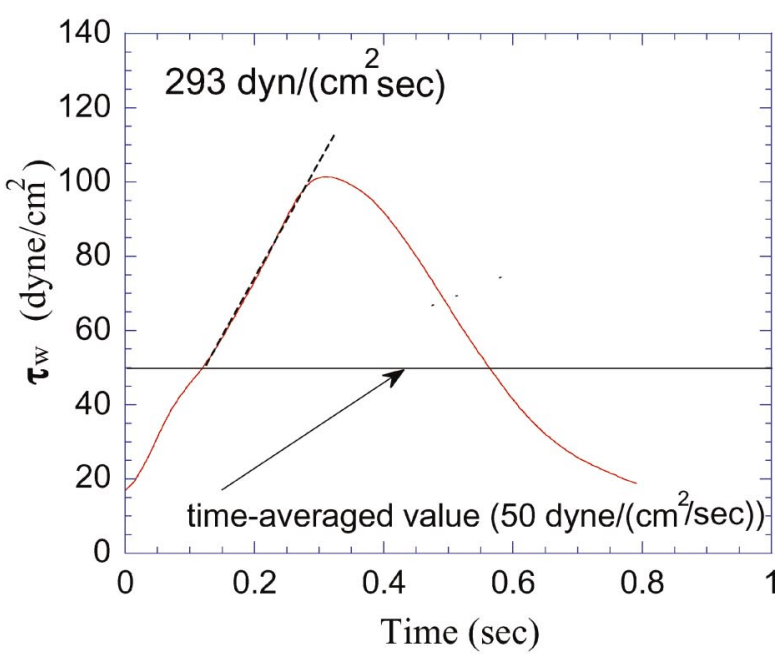

(b)

FIGURE 5. (a) The voltage output representing the pressure difference upstream from the channel was phase-averaged and converted to flow rate for both high and low slew rates. (b) Pulsatile shear stress profiles at $1 \mathrm{~Hz}$ from (a). The linear horizontal line denotes the time-averaged shear stress at $50 \mathrm{dyn} /\left(\mathrm{cm}^{2} \mathrm{~s}\right)$.

response to flow conditions were captured by phase contrast microscope (Nikon Eclipse TE 200), and transmitted by a CCD camera (SONY CCD-IRIS/RGB, Model DXC 151A) to a TV monitor. Imaging data collection spanned $12 \mathrm{~h}$ to visualize different stages of cell-cell interactions and tight association of cell-cell interaction throughout the observation period. Real-time shear stress at which different stages of cell-cell interactions occurred was linked with monocyte-monocyte binding and separation, monocyte tethering and adhesion with the EC monolayers. At a given shear stress, the average velocity of the flow field, which was calculated from Eq. (7), was compared with the individual monocyte velocities. The nonviable monocytes were used as the control data. Nonviable monocytes harbored the similar diameter and density as viable monocytes; however, they lacked the ability to engage in
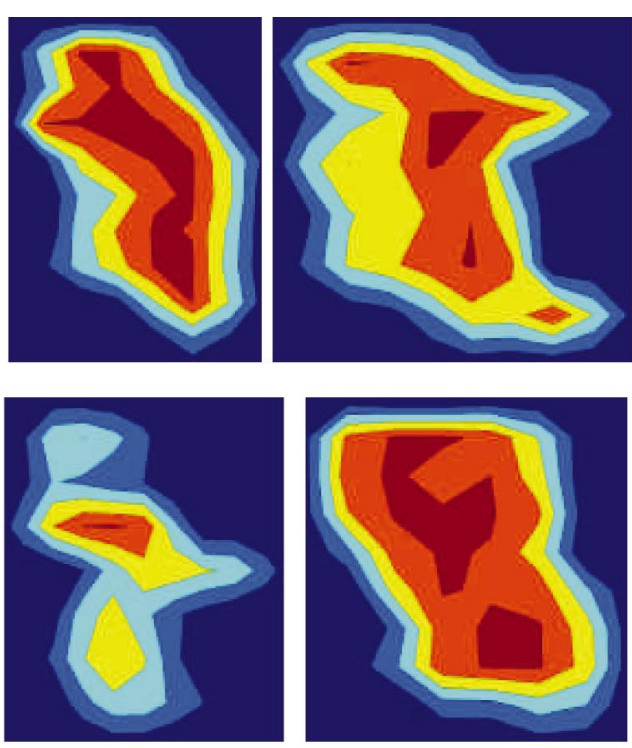

(a)

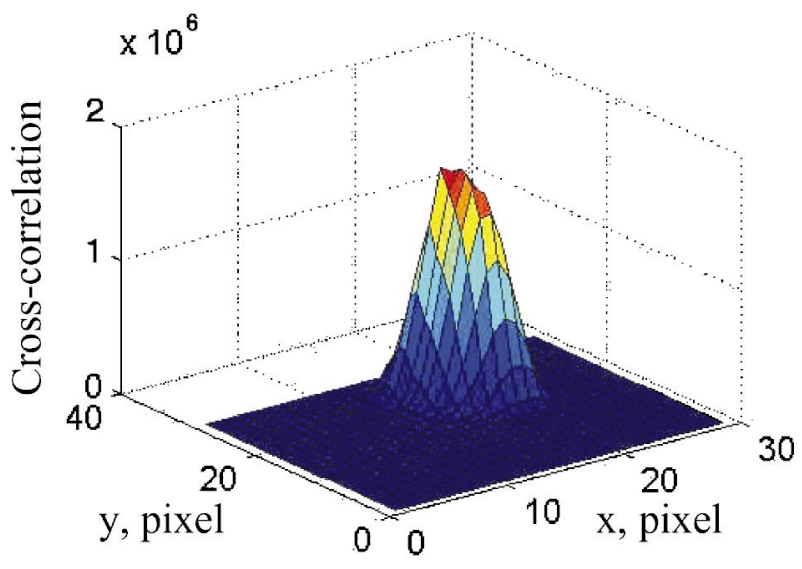

(b)

FIGURE 6. Cell-tracking velocimetry: (a) Intensity contours of monocytes obtained from digitized video images; (b) typical cross-correlation for determining the monocyte displacement.

receptor-integrin interactions with the EC monocytes. These nonviable cells remained afloat at the vagary of shear stress, failing to adhere to the BAEC monolayers. Optically, they harbored ill-defined border morphology under high power field (HPF). Using cell-tracking velocimetry, we compared their trajectories in relation to the flow fields and to the viable monocytes.

\section{Quantitative Real-Time Reverse Transcriptase-Polymerase Chain Reactions (RT-PCR)}

After BAEC were exposed to the flow conditions, total RNA was isolated using RNeasy kit (Qiagen). Real-time RT-PCR was performed according to the 


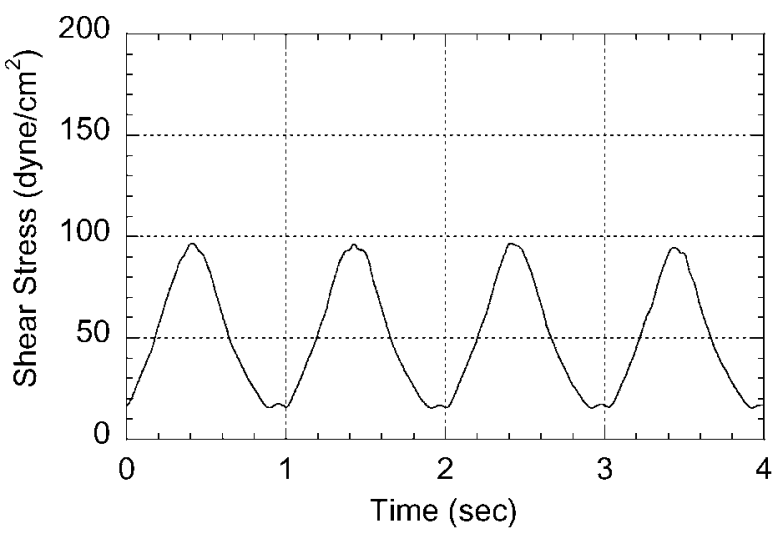

(a)

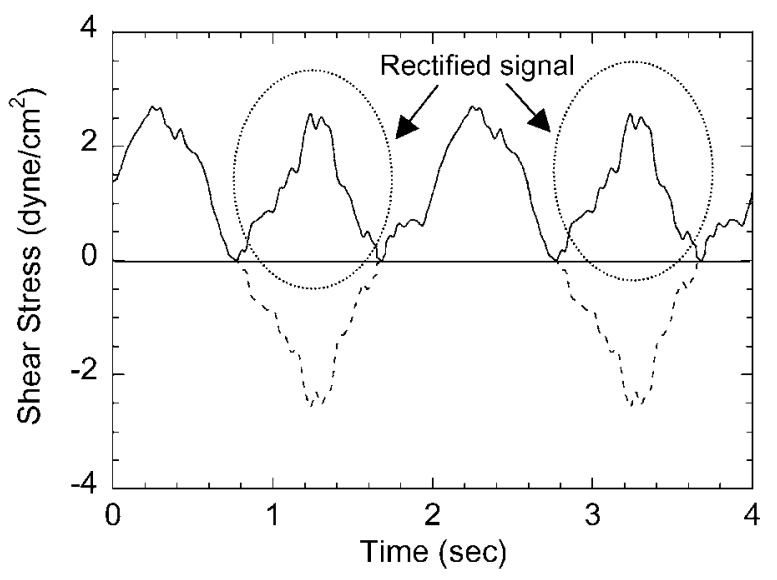

(b)

FIGURE 7. Shear stress measurement by the micro thermal shear stress sensors: (a) pulsatile flow; (b) oscillatory flow to simulate the outer wall of arterial bifurcations at the reattachment point. The rectified signal reflected flow reversal.

recommendations of PE Biosystems TaqMan PCR Core Reagent Kit. ${ }^{2}$ Briefly, equal amounts of RNA at $0.5 \mu \mathrm{g} / \mu \mathrm{l}$ were reverse-transcribed to bring the mixed solution to a final concentration of $1 \times$ TaqMan buffer, $5 \mathrm{mmol} / \mathrm{l}$ $\mathrm{MgCl}_{2}, 200 \mu \mathrm{mol} / \mathrm{l} \mathrm{dATP} / \mathrm{dCTP} / \mathrm{dGTP}, 400 \mu \mathrm{mol} / \mathrm{l} \mathrm{dUTP}$, $100 \mathrm{nmol} / 1 \mathrm{probe}, 400 \mathrm{nmol} / 1$ primers, $0.01 \mathrm{U} / \mu \mathrm{l}$ AmpErase, and $0.025 \mathrm{U} / \mu \mathrm{l}$ AmpliTag Cold DNA polymerase. Total RNA at $0.4 \mathrm{ng} / \mu \mathrm{l}$ in $5 \mu \mathrm{l}$ was then transferred to the 96well plate. PCR were performed at $50^{\circ} \mathrm{C}$ for $2 \mathrm{~min}$ and at $95^{\circ} \mathrm{C}$ for $10 \mathrm{~min}$ and then ran for 40 cycles at $95^{\circ} \mathrm{C}$ for $15 \mathrm{~s}$ and $60^{\circ} \mathrm{C}$ for $1 \mathrm{~min}$ on the real-time RT-PCR Engine (MJ Research Opticon ${ }^{\circledR}$ ). $C_{\mathrm{T}}$, which is the threshold cycle number at which the initial amplification becomes detectable by fluorescence, $\Delta R_{\mathrm{n}}$, defined as normalized fluorescence. TaqMan probes were used for added specificity and sensitivity. ${ }^{37}$ Assuming amplification is $100 \%$ efficient, we used the difference in $C_{\mathrm{T}}$ values for various flow conditions vs. control to mathematically determine the relative difference in the levels of P-Selectin, intercellular adhesion molecule (ICAM-1), and monocyte chemoattractant protein-1 (MCP-1) mRNA expression. ${ }^{23}$ The primers (Table 1) were produced on an automated synthesizer (Applied Biosystems) according to the manufacturer's protocol. For each gene, quantitative RT-PCR was conducted in duplicate. To ensure the quality of the measurements, both negative and positive controls were systematically included in duplicate in each plate. The statistical analysis of the quantitative RT-PCR results was done using the $\Delta C_{\mathrm{T}}$ value ( $C_{\text {Tgene of interest }}-C_{\text {T GAPDH }}$ ). Relative gene expression was obtained by $\Delta \Delta C_{\mathrm{T}}$ methods $\left(\Delta C_{\text {Tsample }}-\Delta C_{\mathrm{T} \mathrm{GAPDH}}\right)$ using the control group as a calibrator for comparison of every unknown sample gene expression level. The conversion between $\Delta \Delta C_{\mathrm{T}}$ and relative gene expression levels is Fold induction $=2^{-\Delta \Delta C_{\mathrm{T}}} \cdot{ }^{21}$

\section{Monocyte Adhesion Assay}

After $4 \mathrm{~h}$ of pulsatile vs. oscillatory flow conditions as described in the Experimental Protocol section, BAEC monolayers on the cover slides were then rinsed with DMEM, and assayed for monocyte-binding activities. Endothelial monolayers were incubated with freshly isolated monocytes $\left(10^{5}\right.$ monocytes $/ \mathrm{cm}^{2}$ ) for $10 \mathrm{~min}$ at $37^{\circ} \mathrm{C}$ under static conditions. Nonadherent monocytes were washed with DMEM. The adherent monocytes were fixed with $1 \%$ glutaraldehyde. Adherent monocytes were counted in a total of 20 high power $(400 \times)$ standardized fields.

\section{Statistics}

The values for shear stress at which monocyte-monocyte binding and separation, monocyte-EC tethering, adhesion, and detachment were expressed as mean values \pm standard deviation. A nonparametric (Wilcoxon rank sum test) comparison of the groups (means of five cell-cell binding events) was applied. $P$ value, which defined the $95 \%$ confident interval, was considered statistically significant at $<0.05$.

\section{RESULTS}

\section{Real-Time Shear Stress by MEMS Sensors}

Two flow profiles representative of different sites at vascular branching points were generated: oscillatory and pulsatile flow. Using the linear calibration curve [Fig. 4(b)], we obtained real-time pulsatile vs. oscillatory shear stresses that were delivered to $\mathrm{EC}$ at $37.0 \pm 0.15^{\circ} \mathrm{C}$ [Figs. 7(a) and $7(\mathrm{~b})]$. The oscillatory shear stress at an arbitrary periodicity of $0.5 \mathrm{~Hz}$ simulated the flow pattern seen at the reattachment points. The time-averaged shear stress $\left(\tau_{\text {ave }}\right)$ was $50 \mathrm{dyn} / \mathrm{cm}^{2}$ for the pulsatile flow. The flow reversal was recorded as upward deflection as rectified signals [Fig. 7(b)]. The pulsatile shear stress measurement 
TABLE 1. Primers used for quantitative real-time RT-PCR.

\begin{tabular}{|c|c|}
\hline Gene & Primer sequences and TaqMan ${ }^{\circledR}$ probes \\
\hline \multirow[t]{3}{*}{ P-selectin } & Forward: 5'-GGGCCACTGACTATCCAGGA-3' \\
\hline & Reverse: 5'-AACCCGTCACTAAGCCTGTTGT-3' \\
\hline & 5'-6(FAM)-ACCCTGACTTATGTTGGTGGAGCAGCA-3' \\
\hline \multirow[t]{3}{*}{ ICAM-1 } & Forward: 5'-CGAGAAGAGAGGACCATGGC-3' \\
\hline & Reverse: 5'-GCCCTTGTGACCGCAGG-3' \\
\hline & 5'-6(FAM)-CCAATTTCTCTTGCCGCTGGGAACTG-3' \\
\hline \multirow[t]{3}{*}{ MCP-1 } & Forward: 5'-GAGGCCAAACCAGAGACCAA-3' \\
\hline & Reverse: 5'-GCAGCGGAGACCTTCATGTT-3' \\
\hline & 5'-6(FAM)-CACGCTGAAACTTGAATCCTCTCGCTG-(TAMRA)3' \\
\hline \multirow[t]{3}{*}{ eNOS } & Forward: 5'-ACGCCGCCACGGTGTCCTTC \\
\hline & Reverse: 3'-GCCCACAAGCTGGATGAGA \\
\hline & 5'-6(FAM)-TCAGAACTTCTTGGAGATTCAGGTT \\
\hline \multirow[t]{3}{*}{ GAPDH } & Forward: 5'-CCCACTCCCAACGTGTCTG'-3' \\
\hline & Reverse: 5'-TCTTTGGACGGTTCATACTACTCTA'3' \\
\hline & TaqMan probe 5'-TGTGGATCTGACCTGCCCGCCTG-3' \\
\hline
\end{tabular}

using the MEMS sensors was compared and validated with the data using the Bernoulli's Principle with an orifice [Fig. 5(b)]. ${ }^{11}$ Both methods generated overlapping shear stress measurement.

The micro shear stress sensor offers three particularly novel design characteristics: (1) the heat insulation features a free standing diaphragm on a vacuum cavity, which minimizes heat loss to the substrate; (2) the semiconductor material serves as a high performance heating/sensing element providing a much higher resistivity than metal which is used in conventional thermal sensors; and (3) the high sensitivity of sensing element allows for real-time shear stress measurements (frequency $>10 \mathrm{kHz}$ ) and a fine spatial resolution comparable to the length of an elongated EC $(<100 \mu \mathrm{m})$. Time constant of sensors was obtained by feeding an electronic square wave into the $E_{\mathrm{t}}$ terminal of the CT circuit. $^{22}$ The transient voltage response was measured at $85 \mu \mathrm{s}$, or $11.7 \mathrm{kHz}$. This high frequency response enabled us to measure oscillating shear stress otherwise difficult with the conventional Bernoulli's Principle using an orifice. $^{11}$

\section{Nonlinear Displacement of Monocyte Locomotion in Response to Oscillatory Flow}

The paths followed by monocyte locomotion were traced by cell-tracking velocimetry, revealing diverse modes of cell-cell interactions in response to oscillating flow. A sequence of images reveals two monocytes undergoing attachment; separation, short-lived reattachment, and reseparation with one another were captured [Figs. 8(a)8(e)]. Both monocytes briefly established adhesion on EC [Fig. 8(b)]. Monocyte 2 then separated from monocyte 1, which eventually established solid adhesion on EC as illustrated at 0 reference point on the $y$-axis [Fig. 8(d)]. The corresponding trajectories of these two individual monocytes were captured using cell-tracking velocimetry
[Fig. 8(f)]. Monocyte 1 was observed to undergo tethering, characterized as abrupt halt in locomotion alternating with resumption of to-and-fro motion. Despite the identical magnitude of oscillatory shear stress, the absolute displacement, velocities, and direction of monocytes were nonlinear, and nonrandom, suggesting the dynamics of molecular interactions underlying the cell-cell binding kinetics. The rolling velocities of individual monocytes were distinct from the average hydrodynamic velocity of the flow field [Fig. 8(g)]. Once in contact with the EC monolayers, the locomotion of individual monocytes was retarded. The trajectory of the nonviable monocytes which were buoyant above the EC monolayer, contrasted those of monocytes 1 and 2 [Fig. 8(f)]. The trajectory of nonviable monocyte followed the direction of shear stress; whereas, the motion of monocytes was influenced by the EC monolayers.

Additional sequence of trajectories shows the meandering paths of three monocytes undergoing to-and-fro rolling and firmed attachment to the EC [Fig. 9(a)]. Under oscillatory shear stress, the irregularities of monocyte trajectory could be due to the undulating surface of the EC monolayers, and the time variation in integrinligand bond formation and breakage. Individual monocytes were observed to display binding and separation with other monocytes, transient tethers and solid attachment to the EC monolayers [Fig. 9(b)]. Figure 10(a) elucidates the velocity profile of a monocyte undergoing tethering in response to oscillating flow at $1 \mathrm{~Hz}$. Figure 10(b) demonstrates two velocity profiles nearly at $0 \mu \mathrm{m} / \mathrm{s}$, suggesting two monocytes establishing firm attachment to BAEC monolayer at $1 \mathrm{~Hz}$. Therefore, the patterns of displacement facilitate the identification of cell-cell binding, monocyte rolling, transient adhesion, and solid adhesion to ECs. The velocity profiles of individual monocytes provide clues to monocytes undergoing dynamic changes in displacement. 

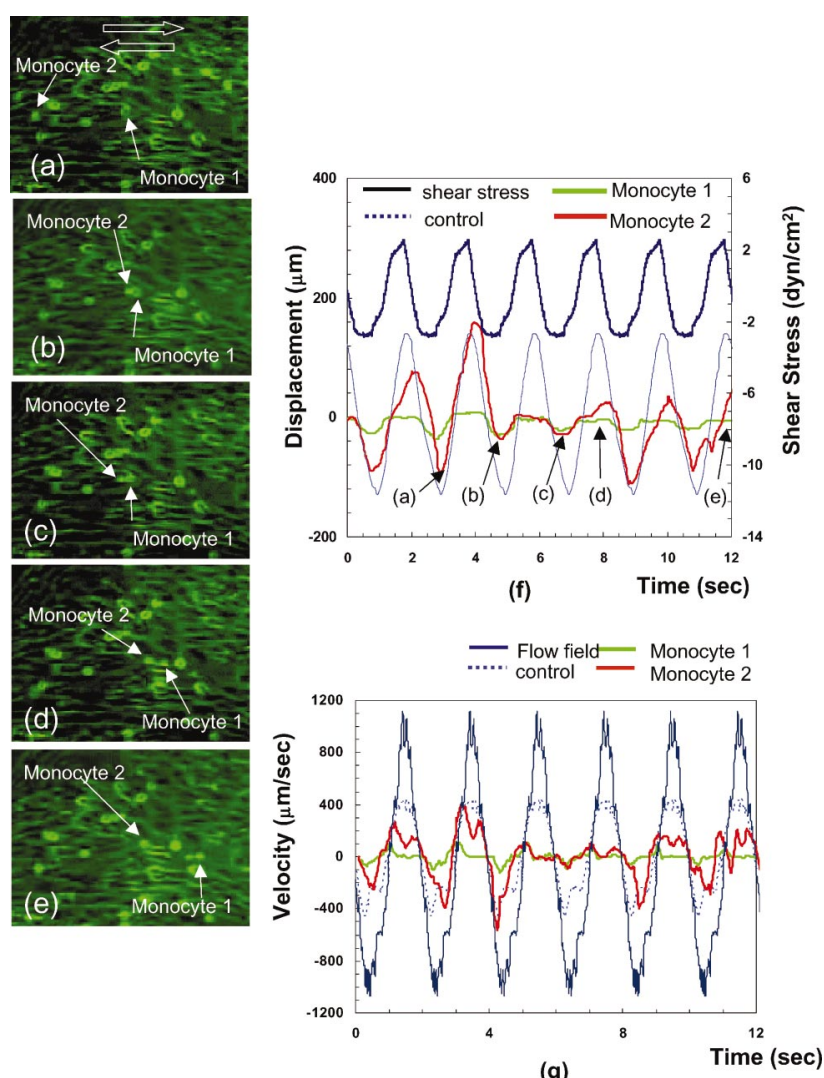

(g)

FIGURE 8. Captured images of cell-cell interactions under oscillatory shear stress: (a) monocyte 1 was undergoing tethering while monocyte 2 displaying to-and-fro locomotion; (b) monocytes 1 and 2 established binding; (c) monocytes 1 and 2 started to separate; (d) monocytes 1 and 2 were apart; (e) monocyte 2 resumed to-and-fro locomotion while monocyte 1 remained attached to EC. (f) The dark blue profile reflects the real-time oscillatory shear stress. The trajectories of monocytes 1 and 2 from the captured images (a-e) are superimposed with the dotted blue trajectory of the nonviable monocyte (control). (g) Velocity profiles of monocytes 1 and 2 are compared with that of the nonviable monocyte (control) in relation to the velocity of oscillating flow field. Theses images were synaptic recordings over $12 \mathrm{~h}$ of oscillatory flow exposure.

\section{Effects of Pulsatile vs. Oscillating Shear Stress on P-Selectin, ICAM-1, and MCP-1 and eNOS mRNA Expression}

The expression of adhesion molecules and chemokines mediated monocyte/EC interactions. Introduction of pulsatile flow with $\partial \tau / \partial t=293 \mathrm{dyn} / \mathrm{cm}^{2} \mathrm{~s}$, and $\tau_{\text {ave }}=50 \mathrm{dyn} / \mathrm{cm}^{2}$ vs. oscillatory flow at $\pm 2.6 \mathrm{dyn} / \mathrm{cm}^{2}$ at $\tau_{\text {ave }}=5$ for $4 \mathrm{~h}$ differentially induced mRNA expression of P-selectin, ICAM-1, and MCP-1 [Fig. 11(a)]. Compared with pulsatile flow, oscillatory flow significantly upregulated P-selectin mRNA expression by 3.4-fold, ICAM-1 by 10.5 -fold, MCP- 1 by 4.8 -fold (oscillatory vs. pulsatile flow: P-selectin: $5.1 \pm 0.63$ vs. $1.75 \pm 0.63$; ICAM-1: 26.5 \pm 3.3 vs. $2.5 \pm 0.7$; MCP- $1: 8.47 \pm 0.63$ vs. $1.75 \pm 0.62$. $P<0.05, n=3$ ) [Fig. 11(b)].

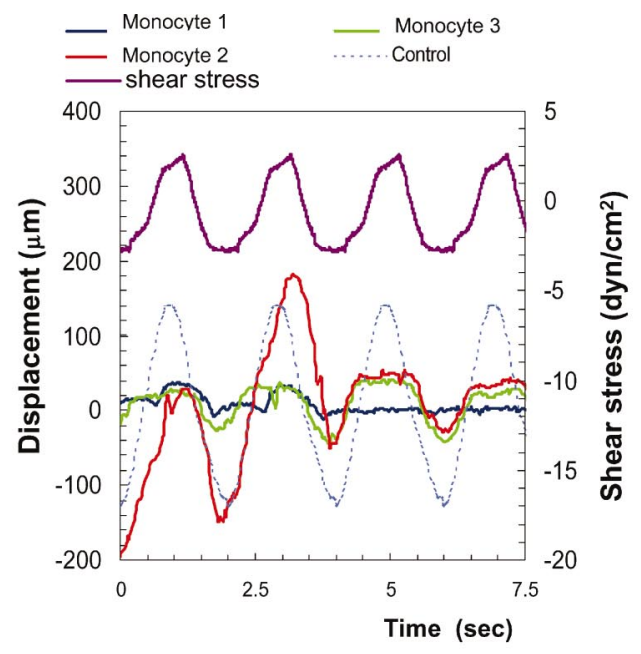

(b)

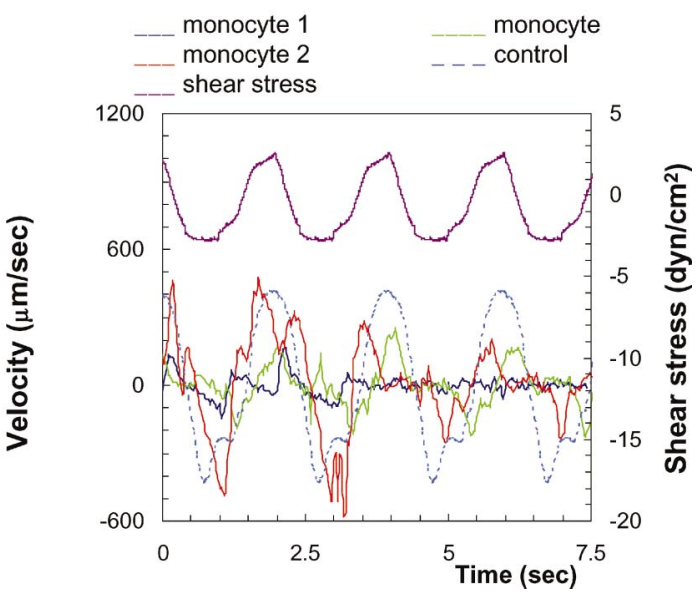

FIGURE 9. (a) Distinct monocyte trajectories illustrate the meandering paths of three monocytes in response to oscillatory shear stress, undergoing to-and-fro rolling, followed by attachment to the EC (monocyte 1) and close interaction between monocytes 2 and 3 . The displacement of nonviable monocyte follows the direction of shear stress. (b) Three distinct velocity profiles of monocytes convey detailed information of binding kinetics compared with the almost harmonic profile of nonviable monocyte. The precise delineation of velocity profiles would be otherwise difficult to capture without the imaging modality. Theses tracings represent synaptic recordings over $12 \mathrm{~h}$ of oscillatory flow exposure.

\section{Monocyte Binding to BAEC in Response to Pulsatile vs. Oscillatory Flow}

After $4 \mathrm{~h}$ of pulsatile vs. oscillatory flow conditions, monocyte adhesion assays were performed. The number of monocyte binding to the BAEC after unidirectional pulsatile flow exposure was significantly attenuated [4 \pm 1 monocytes/high power field (HPF), $P<0.05$ ] In contrast, the distribution and probability of monocytes binding to EC exposed to oscillatory flow was enhanced (static control $=5 \pm 2$ monocytes/HPF, oscillatory flow $=36 \pm 5, P<0.05)$ [Fig. 12]. Hence, the upregulation of adhesion molecule and chemokine expression in response to oscillatory shear stress implicated their roles in recruiting 
(a)

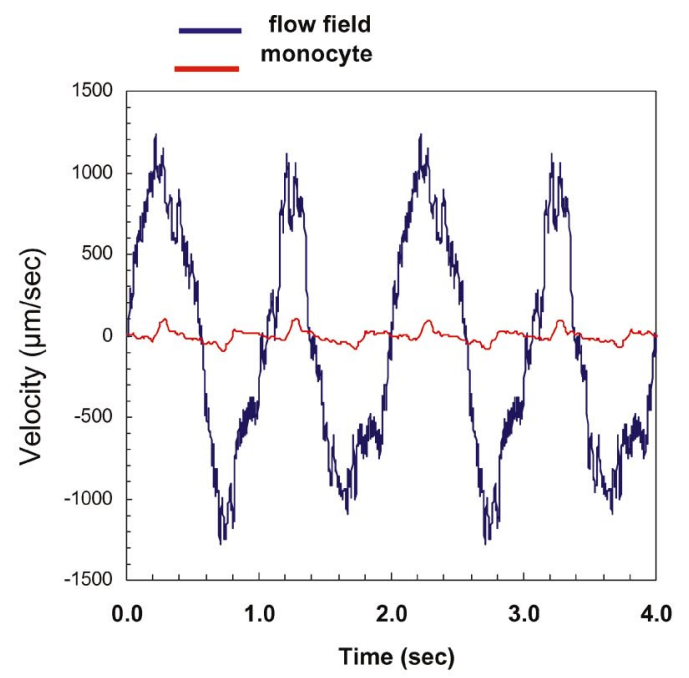

(b)

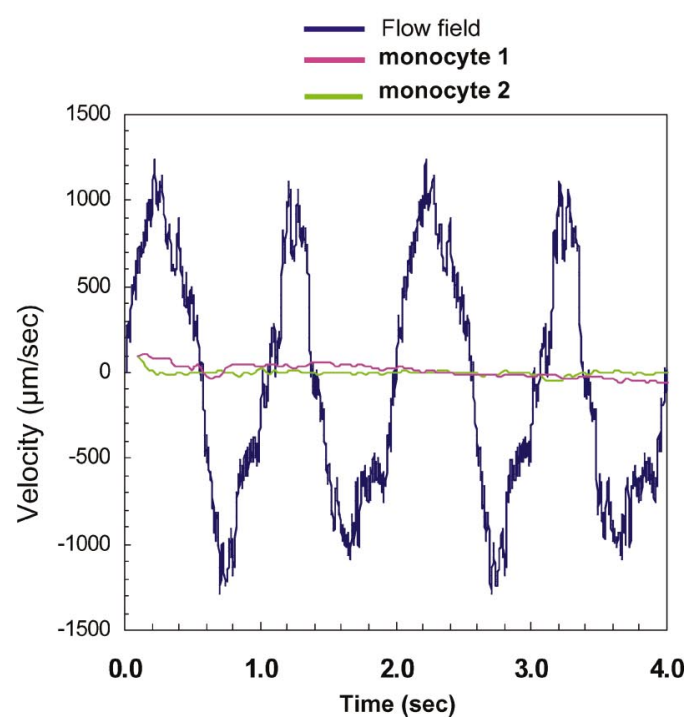

FIGURE 10. (a) Velocity profile of a monocyte undergoing tethering in response to oscillating flow at $1 \mathrm{~Hz}$. The dark blue line represents the average velocity of the flow field. (b) Velocity profiles of two monocytes undergoing attachment to BAEC monolayer in relation to the average velocity in the oscillating flow field at $1 \mathrm{~Hz}$. BAEC monolayers were exposed to oscillatory flow prior to introduction of the monocytes. Theses profiles represent synaptic recordings over $12 \mathrm{~h}$ of oscillatory flow exposure.

monocytes as the initiating events in inflammatory responses.

\section{DISCUSSION}

This report introduces MEMS shear stress sensors and cell-tracking velocity as novel tools for real-time quantification and visualization of monocyte adhesion events. We demonstrated the distinct behaviors of monocyte/EC binding kinetics in response to oscillatory shear stress with high temporal and spatial resolution. The induction of adhesion molecules and chemokines from BAEC in the presence

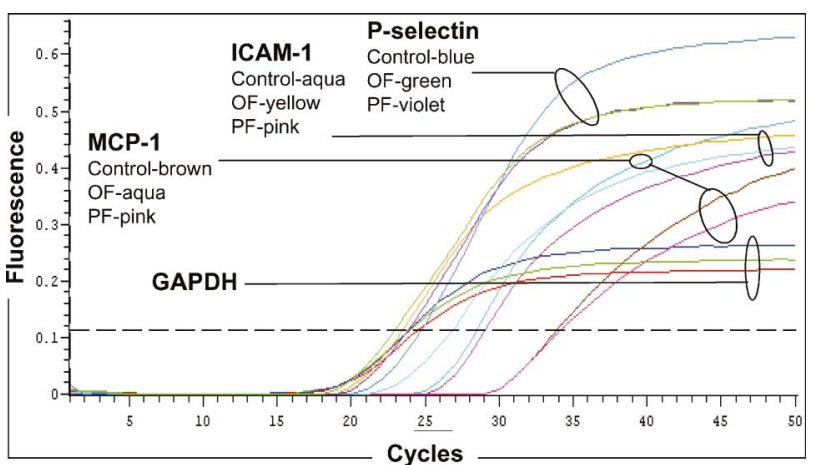

(a)

Rea-Time P-selectin, ICAM-1 and MCP-1 Expression

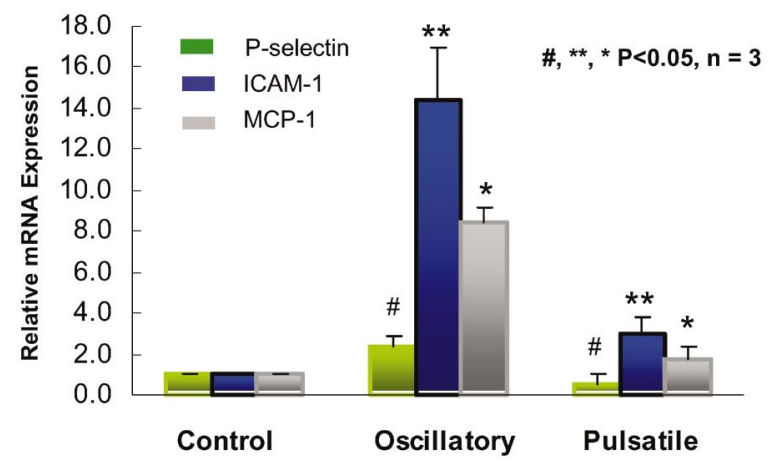

(b)

FIGURE 11. (a) Fluorescence signal vs. cycle number for $P$ selectin, ICAM-1, and MCP-1 normalized with GAPDH. (b) Bar graphs show relative mRNA expression for P-selectin, ICAM-1, MCP-1 in response to pulsatile vs. oscillatory flow conditions at $4 \mathrm{~h}$. Values are expressed as mean \pm SEM.

of an oscillatory shear environment modulated the duration of monocyte tethering, and subsequent attachment. The increase in residence time was conducive to monocyte receptors binding to sufficient ligands such as P-selectin and ICAM-1.

Shear environment in the vasculature provides the dynamics and molecular constituents necessary to mediate leukocyte-EC adhesion. Not only the magnitude but also the temporal component of shear stress is important in regulating the adhesive interactions. The binding kinetics, which is mediated by adhesion molecules such as selectin and integrin, was reported to range from 4 to $7 \mathrm{dyn} / \mathrm{cm}^{2} .{ }^{35}$ Shear stress has been implicated in modulating the kinetics and receptor specificity of polymorphonuclear cell (PMN) - tumor cell interactions. ${ }^{16}$ Furthermore, the kinetics of PMN transmigration through EC junctions was significantly faster than that of PMNs placed under static conditions. ${ }^{18}$

The nature of oscillatory flow, specifically, low mean shear stress and high shear stress gradient, has been shown to induce the expression of inflammatory markers such as ICAM-1, E-selectin, and ET- $1,{ }^{1}$ activation of NADH 


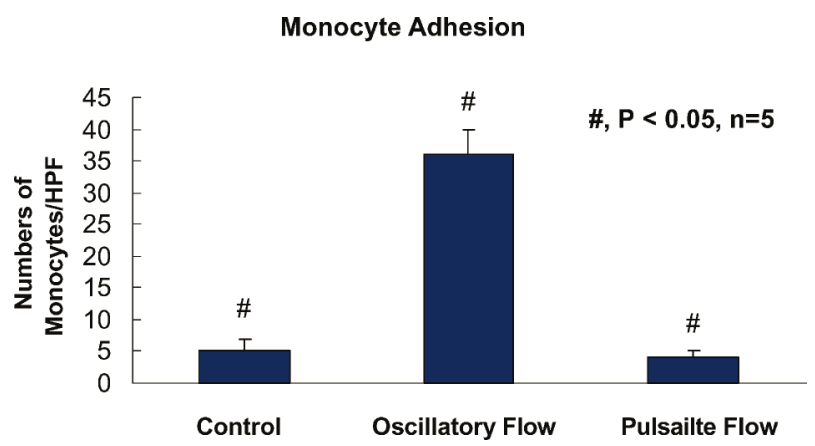

FIGURE 12. Effect of pulsatile vs. oscillatory flow on monocyte binding to BAEC by monocyte adhesion assay at $4 \mathrm{~h}$. Oscillatory flow induced a statistically significant increase in the number of adherent monocytes by 1.2-fold per high power field (HPF) (oscillatory: $36 \pm 4$, control: $5 \pm 2, P<0.05, n=5$ ). Pulsatile flow attenuated the number of monocyte bound by 1.6-fold (pulsatile flow: $4 \pm 1$ ). Data (mean $\pm S D$ ) are based on five separate experiments.

oxidase system, heme oxygenase-1 mRNA, and production of reactive oxygen species. ${ }^{3}$ Recently, Sorescu et al. demonstrated that bone morphogenic protein 4 (BMP4) as a mechanosensitive and pro-inflammatory factor in early steps of atherosclerosis in the arterial regions exposed to oscillatory shear stress. ${ }^{33}$ The upregulation of adhesion molecules and cytokines in response to the oscillatory nature of shear stress $\left(\tau_{\mathrm{ave}}=0\right)$ affected the unique behavior of cell-cell interactions in which a sequence of bond formation-breakage events occurred causing the monocytes to undergo to-and-fro rolling, transient tethering before firm reattachment on the undulating EC surface.

The dynamic visualization in our model displays the nonlinear locomotion of monocytes mediated by the resident time of the oscillatory flow, undulating surface of EC monolayers, integrin-ligand engagement, and expression of chemokines. The use of vitronectin-coated glass rather than more physiologic substrates may affect cellular responses since normal artery wall is compliant and has more complex extracellular matrix. Since EC produces basement membrane in culture, the substrate in this model is reasonably physiologic. The lack of erythrocytes in the medium may also influence the leukocyte-endothelial interactions. Rolling red cells may collide with, enhance binding of, or displace the leukocytes interacting with EC. ${ }^{25,31}$ However, these effects are likely to be small and second-orders. Overall, this modeling provides previously unachievable quantitative analysis. MEMS technology and cell-tracking velocimetry offer an entry point to study monocyte/EC focal contact dynamics and variation in binding kinetics under realistic shear stress fields.

The micro shear stress sensors based on heat-transfer principles were developed by Liu et al. ${ }^{22}$ However, to make MEMS device a reality for biomedical application, we designed the MEMS sensors tailored to the configuration of the pulsatile flow channel under physiologic condition. The micro-circuitry of the sensors was insulated by a $1.5-\mu \mathrm{m}$ layer of oxide by low-pressure chemical vapor deposition to prevent resistance drift from spontaneous oxidation. Backside wiring was established with an IC board.

The shear stress measurement is most sensitive when the span-wise direction of sensors is perpendicular to the flow. The sensitivity of the sensor is proportional to the direction of flow at an angle to the longitudinal direction of the sensing element as $U=U^{*} \cos \theta$. Five-degree misalignment would result in less than $1 \%$ error in velocity measurement. The span-wise length of the resistive element determines the thermal conduction to the substrate; thereby, the sensitivity. The calibration constants, $A$ and $B$, of the linear calibration curves may vary from other sensors because of different fabrication processes and doping concentration [Fig. 3(b)]. However, the difference among individual sensors is likely to be small provided that boron doping into the polysilicon on the same chip is uniform. To avoid thermal interaction between sensors, we will need to incorporate the temperature compensation circuit to the existing constant temperature (CT) mode driving circuit. Xu et al. has developed a circuit that uses another on-chip polysilicon sensor to accomplish the temperature compensation. This sensor has a matched temperature coefficient of resistance (TCR) with the shear stress sensor. ${ }^{38}$

The individual MEMS sensors, which operate on the heat-transfer principles, would not provide the directionality of oscillatory flow. Rectified signals reflect flow reversal from a single sensor [Fig. 7(b)]. Two separately calibrated sensors will be needed to provide the direction of the flow.

The sensitivity of the sensor is also related to the resistance of the sensing element, which, in turn, is dependent on the boron-doping concentration into the polysilicon. This property is related to the frequency response of the hot film as a function of the sensor resistance. What underlies the MEMS sensor's high-frequency response is the elevated resistance $1-10 \mathrm{k} \Omega$ compared with the traditional metal sensors from 5 to $50 \Omega .{ }^{15}$ Previously, Liu et al. reported the measured time constant to be $72 \mu \mathrm{s}$ for the MEMS sensors. ${ }^{22}$ According to the approximate relation between the time constant, $t_{\mathrm{c}}$, and cutoff frequency, $f_{\mathrm{c}}$, at constant temperature, $f_{\mathrm{c}}=1 /\left(1.5 t_{\mathrm{c}}\right)$, the cutoff frequency is estimated to be $9 \mathrm{kHz} .{ }^{14}$ Huang et al. showed that micro shear stress sensor operating in the CT mode can reach a cutoff frequency of $70 \mathrm{kHz}$ and a shear stress sensitivity of $7 \mathrm{mV} /\left(\mathrm{dyn} / \mathrm{cm}^{2}\right) .{ }^{15}$ The sensitivity of our sensors was estimated at $154 \mathrm{mV} /\left(\mathrm{dyn} / \mathrm{cm}^{2}\right)$.

While there are various algorithms for cell-tracking, we have adopted a new code tailored to our pulsatile flow channel. The algorithm was designed to filter the nonuniform background intensity of the digital images from the presence of ECs. The algorithm allowed for tracking several monocytes in the flow fields, displaying different events of cell-cell interactions with various morphology and intensity on the digital images. Our algorithm was developed 
with minimal computational steps. We selected the sampling frequency to identify the monocytes for individual interrogation regions, satisfying the Nyquist frequency. The video capture rate of 30 frames per second was below the range to image instantaneous adhesion events. However, the reconstructed velocity and displacement profiles of individual monocytes allowed for precise recognition of various cell-cell interactions.

\section{CONCLUSION}

Real-time shear stress and cell-tracking velocimetry provide a window into the dynamics of monocyte behaviors in response to oscillatory flow. The visualization of cellcell interactions revealed the nonlinear, and nonrandom binding kinetics of monocytes that were not observable by standard histological cell culture techniques. In response to oscillatory shear stress, the monocyte/EC interactions were influenced by the induction of adhesion molecules and chemokine gradients.

\section{ACKNOWLEDGMENTS}

T.K.H. is supported by grants AHA BGIA (0265166U), NIH Career Development Award (K08 HL068689-01A1), and National Heart Foundation Research Grant (AHAF: H2003-028). This work was also supported by the DARPABioflip Project and by the NIH NRSA \#HL07895.

\section{REFERENCES}

${ }^{1}$ Chappell, D. C., S. E. Varner, R. M. Nerem, R. M. Medford, and R. W. Alexander. Oscillatory shear stress stimulates adhesion molecule expression in cultured human endothelium. Circ. Res. 82:532-539, 1998.

${ }^{2}$ Chu, Y., D. D. Heistad, K. L. Knudtson, K. G. Lamping, and F. M. Faraci. Quantification of mRNA for endothelial NO synthase in mouse blood vessels by real-time polymerase chain reaction. Arterioscler. Thromb. Vasc. Biol. 22:611-616, 2002.

${ }^{3}$ De Keulenaer, G. W., D. C. Chappell, N. Ishizaka, R. M. Nerem, R. W. Alexander, and K. K. Griendling. Oscillatory and steady laminar shear stress differentially affect human endothelial redox state: Role of a superoxide-producing NADH oxidase. Circ. Res. 82:1094-1101, 1998.

${ }^{4}$ DePaola, N., M. A. Gimbrone, Jr., P. F. Davies, and C. F. Dewey, Jr. Vascular endothelium responds to fluid shear stress gradients [published erratum appears in Arterioscler. Thromb. 13:465, 1993]. Arterioscler Thromb. 12:1254-1257, 1992.

${ }^{5}$ Fogelman, A. M., F. Elahi, K. Sykes, B. J. Van Lenten, M. C. Territo, and J. A. Berliner. Modification of the Recalde method for the isolation of human monocytes. J. Lipid Res. 29:12431247,1988

${ }^{6}$ Frangos, J. A., T. Y. Huang, and C. B. Clark. Steady shear and step changes in shear stimulate endothelium via independent mechanisms - superposition of transient and sustained nitric oxide production. Biochem. Biophys. Res. Commun. 224:660$665,1996$.
${ }^{7}$ Fung, Y. C., and S. Q. Liu. Elementary mechanics of the endothelium of blood vessels. J Biomech Eng 115:1-12, 1993.

${ }^{8}$ Glagov, S., C. Zarins, D. P. Giddens, and D. N. Ku. Hemodynamics and atherosclerosis. Insights and perspectives gained from studies of human arteries. Arch. Pathol. Lab. Med. 112:1018-1031, 1988.

${ }^{9}$ Haritonnidis, J. H. The Measurement of Shear Stress in Fluid Mechanics Measurements. Berlin: by Springer-Verlag EMGe-H, 1989, pp. 229-236.

${ }^{10}$ Helmlinger, G., B. C. Berk, and R. M. Nerem. Calcium responses of endothelial cell monolayers subjected to pulsatile and steady laminar flow differ. Am. J. Physiol. 269:C367-C375, 1995.

${ }^{11}$ Hsiai, T., S. K. Cho, S. Hama, M. Navab, L. L. Demer, and C. M. Ho. Endothelial cell dynamics under pulsating flow: Significance of high- vs. low shear stress slew rates. Ann. Biomed. Eng. 30:646-656, 2002.

${ }^{12}$ Hsiai, T., M. Ing, M. Navab, S. Reddy, and C. M. Ho. Microsensors to characterize shear stress regulating inflammatory responses in the arterial bifurcations. In Proceedings of the Second Joint Meeting of the IEEE Engineering in Medicine and Biology and the Biomedical Engineering Society, September 2002, Huston, USA, 20, pp. 662-663.

${ }^{13}$ Hsiai, T. K., S. K. Cho, S. Reddy, S. Hama, M. Navab, L. L. Demer, H. M. Honda, and C. M. Ho. Pulsatile flow regulates monocyte adhesion to oxidized lipid-induced endothelial cells. Arterioscler. Thromb. Vasc. Biol. 21:1770-1776, 2001.

${ }^{14}$ Huang, J., C. M. Ho, S. Tung, C. Liu, and Y. C. Tai. Microthermal shear stress sensor with and without cavity underneath. In: IEEE Instrumentation and Measurement Conference, Waltham, MA, April 1995, pp. 171-174.

${ }^{15}$ Huang, J. B., S. Tung, C. M. Ho, C. Liu, and Y. C. Tai. IEEE Trans. an Instrumentation and Measurement, Vol. 45, No. 2, pp. 570-574, April 12, 1996.

${ }^{16}$ Jadhav, S., B. S. Bochner, and K. Konstantopoulos. Hydrodynamic shear regulates the kinetics and receptor specificity of polymorphonuclear leukocyte-colon carcinoma cell adhesive interactions. J. Immunol. 167:5986-5993, 2001.

${ }^{17}$ King, R. M., and D. A. Hammer. Multiparticle adhesive dynamics: Hydrodynamic recruitment of rolling leukocytes. Proc. Natl. Acad. Sci. 98:14919-14924, 2001.

${ }^{18}$ Kitayama, J., A. Hidemura, H. Saito, and H. Nagawa. Shear stress affects migration behavior of polymorphonuclear cells arrested on endothelium. Cell Immunol. 203:39-46, 2000.

${ }^{19} \mathrm{Ku}$, D. N. Blood flow in arteries. Annu. Rev. Fluid Mech. 29:399-434, 1997.

${ }^{20} \mathrm{Ku}$, D. N., D. P. Giddens, C. K. Zarins, and S. Glagov. Pulsatile flow and atherosclerosis in the human carotid bifurcation. Positive correlation between plaque location and low oscillating shear stress. Arteriosclerosis 5:293-302, 1985.

${ }^{21}$ Lavik, K. J., and T. D. Schmittgen. Analysis of relative gene expression data using real-time quantitative PCR and the 2-(dealta)(dalta)Ct Method. Methods 25:402-408, 2001.

${ }^{22}$ Liu, C., J. B. Huang, Z. Zhu, F. Jiang, S. Tung, Y.-C. Tai, and C.-M. Ho. A micromachined flow shear-stress sensor based on thermal transfer principles. J. MEMS 8:90-99, 1999.

${ }^{23}$ Livak, K. J., and T. D. Schmittgen. Analysis of relative gene expression data using real-time quantative PCR and the 2deltadealtaCT Method. Methods 25, 2001.

${ }^{24}$ Malek, A. M., S. L. Alper, and S. Izumo. Hemodynamic shear stress and its role in atherosclerosis. JAMA 282:2035- 2042, 1999. 
${ }^{25}$ Melder, R. J., J. Yuan, L. L. Munn, and R. K. Jain. Erythrocytes enhance lymphocyte rolling and arrest in vivo. Microvasc. Res. 59:316-322, 2000.

${ }^{26}$ Motomiya, M., and T. Karino. Flow patterns in the human carotid artery bifurcation. Stroke 15:50-56, 1984.

${ }^{27}$ Pedersen, E., M. Agerbaek, I. Kristensen, and A. Yoganathan. Wall shear stress and early atherosclerotic lesions in the abdominal aorta in young adults. Eur. J. Vasc. Endovasc. Surg. 13:443-451, 1997.

${ }^{28}$ Raffel, M., C. Willert, and J. Kompenhans. Particle Image Velocimetry, A Practical Guide. Berlin: Springer-Verlag, 1998.

${ }^{29}$ Ravensbergen, J., J. W. Ravensbergen, J. K. Krijger, B. Hillen, and $\mathrm{H}$. W. Hoogstraten. Localizing role of hemodynamics in atherosclerosis in several human vertebrobasilar junction geometries. Arterioscler. Thromb. Vasc. Biol. 18:708-716, 1998.

${ }^{30}$ Ross, R. Atherosclerosis is an inflammatory disease. Am. Heart. J. 138:S419-S420, 1999.

${ }^{31}$ Schmid-Schonbein, G. W., S. Usami, R. Skalak, and S. Chien. The interaction of leukocytes and erythrocytes in capillary and postcapillary vessels. Microvasc. Res. 19:45-70, 1980.

${ }^{32}$ Shanmugan, K., and A. Breipohl. Random Signals: Detection, Estimation and Data Analysis. New York: Wiley, 1988.

${ }^{33}$ Sorescu, G. P., M. W. D. Sykes, M. O. Platt, A. H. J. Saha, N. L. Boyd, Y. C. Boo, J. D. Vega, W. R. Taylor, and H. Jo. Bone morphogenic protein 4-produced in endothelial cells by oscillatory shear stress stimulates an inflammatory response. $J$. Biol. Chem. 2003, Aug 15;278(33);31128-31135.

${ }^{34}$ Steinman, D. A., J. B. Thomas, H. M. Ladak, J. S. Milner, B. K. Rutt, and J. D. Spence. Reconstruction of carotid bifurcation hemodynamics and wall thickness using computational fluid dynamics and MRI. Magn. Reson. Med. 47:149-159, 2002.

${ }^{35}$ Taylor, A. D., S. Neelamegham, J. D. Hellums, C. W. Smith, and S. I. Simon. Molecular dynamics of the transition from Lselectin- to beta 2-integrin-dependent neutrophil adhesion under defined hydrodynamic shear. Biophys. J. 71:3488-3500, 1996.

${ }^{36}$ Traub, O., and B. C. Berk. Laminar shear stress: Mechanisms by which endothelial cells transduce an atheroprotective force. Arterioscler. Thromb. Vasc. Biol. 18:677-685, 1998.

${ }^{37}$ Walker, N. J. A technique whose time has come. Science 296:557-559, 2002.

${ }^{38} \mathrm{Xu}$, Y., F. Jiang, Q. Lin, J. Clendenen, S. Tung, and Y. C. Tai. Underwater shear stress sensor. In: Fifteenth IEEE International Conference on Micro Electro Mechanical Systems (MEMS '02), Las Vegas, NV, Jan. 20-24, 2002.

${ }^{39}$ Zarins, C. K., D. P. Giddens, B. K. Bharadvaj, V. S. Sottiurai, and R. F. Mabon. Carotid bifurcation of plaque localization with flow velocity profiles and wall shear stress. Circ. Res. 53:502$514,1983$. 